\title{
Boron-Nitride Nanotubes as Zeptogram-Scale Bionanosensors: Theoretical Investigations
}

\author{
R. Chowdhury and S. Adhikari
}

\begin{abstract}
Boron-nitride nanotubes (BNNTs) are being increasingly used due to their superior biocompatibility. We develop sensor equations for using the BNNTs as possible mass sensors for nanoscale biological objects. Two approaches based on static and dynamic deflection shapes have been investigated. Two types of sensor configurations, namely, cantilevered and bridged, have been studied. New sensor equations have been validated using molecular mechanics simulations. Our results show that BNNTs can be used as biosensor with sensitivity reaching in the order of $0.1 \mathrm{zg} / \mathrm{GHz}$.
\end{abstract}

Index Terms-Boron-nitride nanotubes (BNNTs), frequency shift, mass sensor, molecular mechanics.

\section{INTRODUCTION}

C ARBON nanotubes (CNTs) [1] have received remarkable research interests due to their excellent electronic [2], thermal [3], and structural/mechanical [4]-[8] properties. Recently, the usefulness of CNTs in biological applications such as biosensor devices have been realized and numerous advancements have been made in a wide range of application areas [9]-[14]. Various approaches such as CNT field-effect-transistor-based biosensors [12] and cantilever CNTs biosensors [10] have been explored to integrate the CNTs into modern bionanotechnology.

Soon after the discovery of CNTs, Blase et al. [15] theoretically predicted the possibility of obtaining boron-nitride nanotubes (BNNTs). They initiated an investigation on their distinctive properties [16], [17] by exploring the similarity between hexagonal boron-nitride (h-BN) sheets [18] and graphite. An h-BN sheet is composed of alternating atoms of boron and nitrogen, but $\mathrm{BN}$ is mostly found in the same phases, which produce atomic structures similar to those of graphite. It is noted that even the crystallographic parameters of h-BN sheets and graphite are almost equal. Rolling up an $\mathrm{h}-\mathrm{BN}$ sheet in different chiral directions generates BNNTs of various chralities, such as zigzag, chiral, and armchair. Such BNNTs possess many of the superior properties of the CNTs [19], [20] such as exceptional

Manuscript received April 6, 2010; revised July 14, 2010; accepted July 15, 2010. Date of publication July 26, 2010; date of current version July 8, 2011. The work of R. Chowdhury was supported by the Royal Society through the Award of Newton International Fellowship. The work of S. Adhikari was supported by The Leverhulme Trust for the Award of the Philip Leverhulme Prize and The Royal Society of London through the Woulfson Research Merit Award. The review of this paper was arranged by Associate Editor L. Dong.

The authors are with the Multidisciplinary Nanotechnology Centre, Swansea University, Swansea, SA2 8PP, U.K. (e-mail: R.Chowdhury@swansea.ac.uk; S.Adhikari@swansea.ac.uk).

Color versions of one or more of the figures in this paper are available online at http://ieeexplore.ieee.org.

Digital Object Identifier 10.1109/TNANO.2010.2060492 elastic properties [21]-[27], high mechanical strength [28]-[33], chemical inertness [34], structural stability [35], [36], high heat conduction, and piezoelectricity [37]. In addition, BNNT has a wideband gap independent of the geometrical/atomic configuration [19]. These factors make BNNT particularly suitable for the biological applications [38]. Moreover, BNNT is a nonabsorption material of visible and infrared light [38]. This property would particularly help to protect biofragments from overheating and damage, which may be possible in the case of CNT usage.

Several attempts have been made to explore the application of CNTs in biotechnology, while the use of BNNTs have not been elucidated largely [38]. Up till now, a number of investigations have been reported on the use of BNNTs as nanosensor [39]. Ciofani et al. [36] exploited the use of BNNTs in the nanomedicine field. Experimental studies suggest that BNNTs are more suitable for the development of sensors and transducers for the detection of biological entities, due to their chemical stability [36]. Ciofani et al. [39] performed a study on the interactions and effects of biological objects with BNNTs. In a later study, Ciofani et al. [40] highlighted the complexity of BNNTbased nanomaterials, which requires further experiments for the accurate assessment of their biocompatibility. Basic idea of developing nanosensor devices are founded on either the shift in frequency [10], [41]-[43] or the change in conductance [44], [45]. Here, we develop a methodology for the active use of BNNTs as a biosensor, by which we can detect the amount of deposited biomass [10]. We exploited the shift in the resonance frequency due to the added mass on the BNNT resonator [28], [46], [47]. Two types of sensor configurations, namely, cantilevered and bridged, have been studied. Explicit closed-form expressions of the sensitivities of the BNNT sensors have been derived using the continuum-beam theory. New sensor equations have been validated using molecular mechanics simulations.

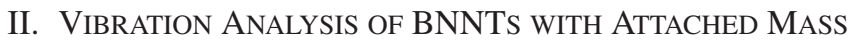

We use Euler-Bernoulli beam theory [48] to model the bending vibration of single-walled BNNT resonators. The continuum models based on beam as well as shell have been used extensively for single and multiwalled CNTs, see, for example, [49]-[51]. This motivates us to use the continuum model to develop sensor equations. The equation of motion of free vibration can be expressed as

$$
E I \frac{\partial^{4} u(x, t)}{\partial x^{4}}+\rho A \frac{\partial^{2} u(x, t)}{\partial t^{2}}=0
$$

where $u(x, t)$ is the transverse deflection, $E$ the Young's modulus, $I$ the second moment of the cross-sectional area $A$, and 
$\rho$ is the density of BNNTs. Depending on the boundary condition of the BNNT and the location of the attached mass, the resonant frequency of the combined system can be derived. We only consider the fundamental resonant frequency, which can be expressed as

$$
f_{n}=\frac{1}{2 \pi} \sqrt{\frac{k_{\mathrm{eq}}}{m_{\mathrm{eq}}}}
$$

where $k_{\mathrm{eq}}$ and $m_{\mathrm{eq}}$ are equivalent stiffness and mass of BNNT with attached mass in the first mode of vibration, respectively. Two kinds of end constraints, i.e., cantilever and bridged, are considered. In the previous study [13], the authors used static deflections to obtain the equivalent stiffness and mass of CNTbased resonators. Here, deflection shapes based on dynamic equation are used for the BNNT resonators. The sensor equations obtained using this approach is expected to have more accurate sensing capabilities as the inertial properties of the BNNTs are taken into account in a more rigorous manner.

\section{A. Cantilevered BNNT With a Mass at the Tip}

Consider that a BNNT resonator of length $L$ is perturbed by a mass $M$ at the end point, i.e., at $x=L$ as shown in cantilever. For the cantilevered BNNT, the resonance frequencies can be obtained from

$$
f_{j}=\frac{\lambda_{j}^{2}}{2 \pi} \sqrt{\frac{E I}{\rho A L^{4}}}
$$

where $\lambda_{j}$ can be obtained by [52] solving the following transcendental equation

$$
\cos \lambda \cosh \lambda+1=0 \text {. }
$$

The vibration mode shape can be expressed as

$$
\begin{aligned}
U_{j}(x)= & \left(\cosh \lambda_{j} \frac{x}{L}-\cos \lambda_{j} \frac{x}{L}\right) \\
& -\left(\frac{\sinh \lambda_{j}-\sin \lambda_{j}}{\cosh \lambda_{j}+\cos \lambda_{j}}\right)\left(\sinh \lambda_{j} \frac{x}{L}-\sin \lambda_{j} \frac{x}{L}\right)
\end{aligned}
$$

The mode shape in (5) is normalized such that

$$
\frac{1}{L} \int_{0}^{L} U_{j}^{2}(x) \mathrm{d} x=1 .
$$

We aim to obtain an equivalent single-DOF (SDOF) oscillator for the mass loaded BNNT in Fig. 1. The equivalence between the actual mass loaded BNNT and the virtual SDOF oscillator can be established be equating the potential and kinetic energies of both the systems. One needs to consider a deflection shape for the BNNT. Since the cantilevered BNNT is vibrating in the first mode, it is natural to consider the deflection proportional to the fist mode of vibration given by (5) with $\lambda_{1}=1.8751$. This value is obtained [53] by solving the transcendental (4). Therefore, the assumed deflection is

$$
U(x)=a U_{1}(x)
$$

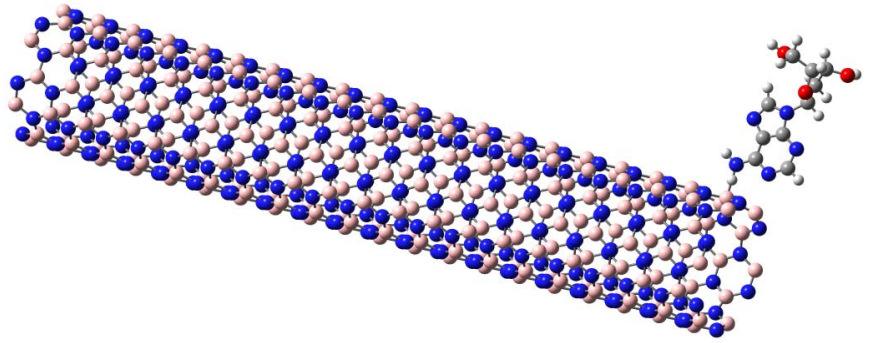

(a)

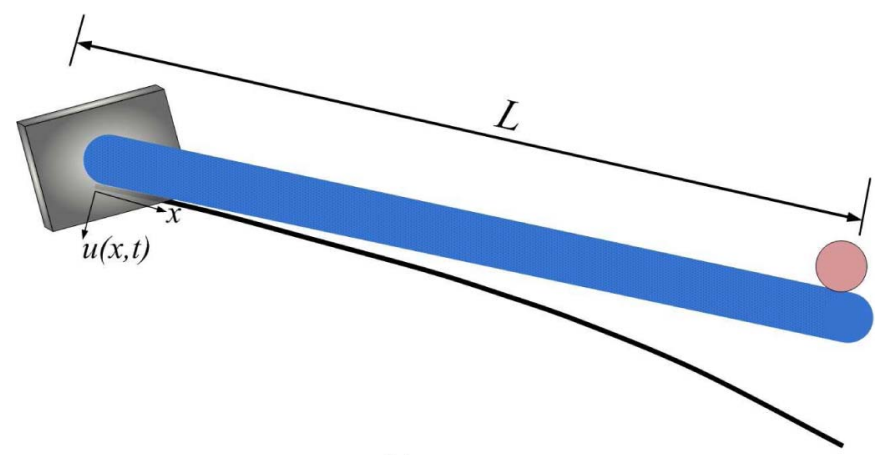

(b)

Fig. 1. Cantilevered BNNT resonator of length $L$ with an attached mass. The mode-shape in the first mode of vibration given by (5) is shown in (b). The maximum deflection occurs under the added mass at $x=L$. (a) DeOxy adenosine with free residue at the edge of a BNNT. (b) Mathematical idealization: point mass at the tip.

where $a$ is a constant amplitude. The potential energy of the BNNT can be obtained as

$P=\frac{1}{2} E I \int_{0}^{L}\left\{\frac{\partial^{2} U(x)}{\partial x^{2}}\right\}^{2} \mathrm{~d} x=\frac{1}{2} E I a^{2} \int_{0}^{L}\left\{\frac{\partial^{2} U_{1}(x)}{\partial x^{2}}\right\}^{2} \mathrm{~d} x$.

Differentiating $U_{j}(x)$ in (5) twice with respect to $x$ and using $\lambda_{1}=1.8751$, this integral can be obtained as

$$
P=12.36236338 a^{2} \frac{E I}{L^{3}} .
$$

Since the potential energy of the equivalent SDOF oscillator is $(1 / 2) k_{\mathrm{eq}} a^{2}$, from the previous equation, we have

$$
k_{\mathrm{eq}}=12.36236338 \frac{E I}{L^{3}} .
$$

Suppose the value of the added mass is $M$. Assuming harmonic motion, i.e., $u(x, t)=U(x) \exp (i \omega t)$, where $\omega$ is the frequency, the kinetic energy of the BNNT can be obtained as

$$
\begin{aligned}
T & =\frac{\omega^{2}}{2} \int_{0}^{L} \rho A U^{2}(x) d x+\frac{\omega^{2}}{2} M U^{2}(L) \\
& =\frac{\omega^{2} a^{2}}{2} \rho A L+\frac{\omega^{2} a^{2}}{2} M(2)^{2}=\frac{\omega^{2} a^{2}}{2}(\rho A L+4 M) .
\end{aligned}
$$

Since the kinetic energy of the equivalent SDOF oscillator is $(1 / 2) m_{\mathrm{eq}} \omega^{2} a^{2}$, we have

$$
m_{\mathrm{eq}}=\rho A L+4 M .
$$


The resonant frequency can be obtained using (2) as

$$
\begin{aligned}
f_{n} & =\frac{1}{2 \pi} \sqrt{\frac{k_{\mathrm{eq}}}{m_{\mathrm{eq}}}}=\frac{1}{2 \pi} \sqrt{\frac{12.36236338\left(E I / L^{3}\right)}{\rho A L+4 M}} \\
& =\frac{1}{2 \pi} \sqrt{12.36236338} \sqrt{\frac{E I}{\rho A L^{4}}} \sqrt{\frac{1}{1+(M / \rho A L) 4}} \\
& =\frac{1}{2 \pi} \frac{\alpha_{k} \beta}{\sqrt{1+\alpha_{m} \Delta M}}
\end{aligned}
$$

where

$$
\begin{aligned}
\alpha_{k} & =\sqrt{12.36236338}=3.516015270 \\
\beta & =\sqrt{\frac{E I}{\rho A L^{4}}} \\
\Delta M & =\frac{M}{\rho A L} \\
\text { and } \quad \alpha_{m} & =4
\end{aligned}
$$

We call $\Delta M$ the relative mass change.

The resonant frequency for a cantilevered BNNT without any added mass is obtained by substituting $\Delta M=0$ in (13) as

$$
f_{0_{n}}=\frac{1}{2 \pi} \alpha_{k} \beta
$$

Comparing this with (3), we indeed obtain $\alpha_{k}=\lambda_{1}^{2}=$ 3.516015270 . This verifies the energy approach adopted here. Next, we extend this analysis to bridged BNNT.

\section{B. Bridged BNNT With a Mass at the Midpoint}

For the bridged BNNT shown in Fig. 2, the equation of motion and the natural frequency equation are given by (1) and (2), respectively. However, the constant $\lambda_{j}$ should obtained [52] by solving

$$
\cos \lambda \cosh \lambda-1=0 \text {. }
$$

The vibration mode shape can be expressed as

$$
\begin{aligned}
U_{j}(x)= & \left(\cosh \lambda_{j} \frac{x}{L}-\cos \lambda_{j} \frac{x}{L}\right) \\
& -\left(\frac{\cosh \lambda_{j}-\cos \lambda_{j}}{\sinh \lambda_{j}-\sin \lambda_{j}}\right)\left(\sinh \lambda_{j} \frac{x}{L}-\sin \lambda_{j} \frac{x}{L}\right) .
\end{aligned}
$$

For the first mode of vibration, we have $\lambda_{1}=4.7300$. This value is obtained [53] by solving the frequency (19). Like the cantilevered case, the mode shape is normalized according to (6).

We assume the deflection shape as in (7) with $U_{1}(x)$ given by (20) for $j=1$. From the potential energy of the BNNT, the equivalent stiffness can be obtained as

$$
k_{\mathrm{eq}}=E I \int_{0}^{L}\left\{\frac{\partial^{2} U_{1}(x)}{\partial x^{2}}\right\}^{2} \mathrm{~d} x=500.5638988 \frac{E I}{L^{3}} .
$$

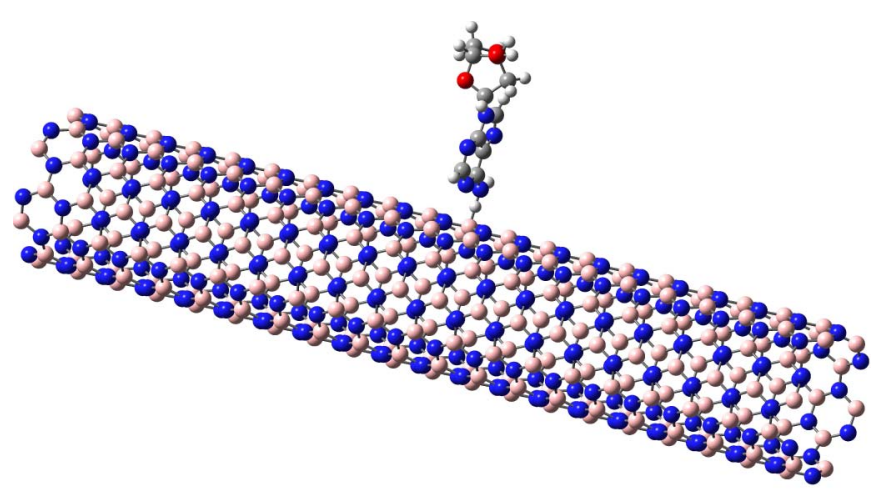

(a)

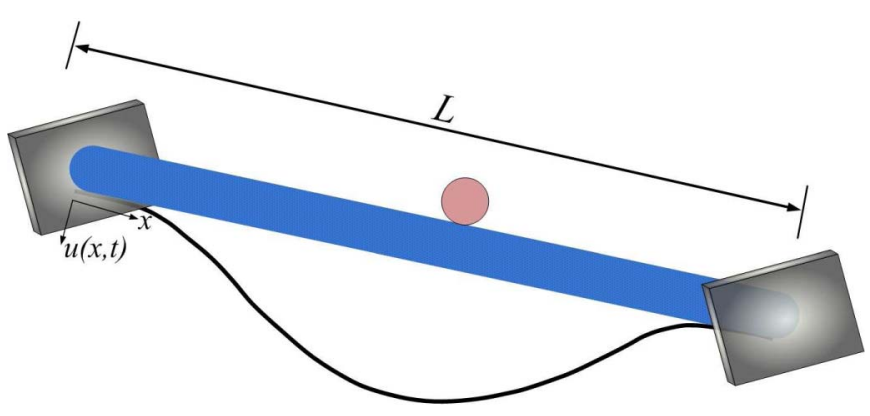

(b)

Fig. 2. Bridged BNNT resonator of length $L$ with an attached mass. The modeshape in the first mode of vibration given by (20) is shown in (b). The maximum deflection occurs under the added mass at $x=L / 2$. (a) DeOxy adenosine with free residue at the center of a BNNT. (b) Mathematical idealization: point mass at the center.

As can be seen in Fig. 2, the value of the added mass at the center of the BNNT is $M$. Using the expressions of the mode shape in (20), the kinetic energy of the bridged BNNT can be obtained as

$$
\begin{aligned}
T & =\frac{\omega^{2}}{2} \int_{0}^{L} \rho A U^{2}(x) d x+\frac{\omega^{2}}{2} M U^{2}\left(\frac{L}{2}\right) \\
& =\frac{\omega^{2} a^{2}}{2}(\rho A L+2.522208550 M) .
\end{aligned}
$$

Therefore, the equivalent mass is

$$
m_{\mathrm{eq}}=\rho A L+2.522208550 M .
$$

The resonant frequency can be obtained using (2) as

$$
f_{n}=\frac{1}{2 \pi} \sqrt{\frac{12.36236338 E I / L^{3}}{\rho A L+2.522208550 M}}=\frac{1}{2 \pi} \frac{\alpha_{k} \beta}{\sqrt{1+\alpha_{m} \Delta M}}
$$

where $\beta$ and $\Delta M$ are as defined in (15) and (16), respectively, and the constants

$$
\begin{aligned}
\alpha_{k} & =\sqrt{500.5638988}=22.37328537 \\
\text { and } \quad \alpha_{m} & =2.522208550 .
\end{aligned}
$$

The resonant frequency for a bridged BNNT without any added mass can be obtained by substituting $\Delta M=0$ in (24) as in (18). Comparing (18) with (3), one obtains $\alpha_{k}=\lambda_{1}^{2}=22.37328537$, which verifies the energy approach adopted here. 


\section{Mass Detection And Sensitivity Calculation}

The expressions of the natural frequencies of the mass loaded BNNT are now used to obtain the mass based on the frequency shift [10]-[14], [42], [54], [55]. Combining (13) and (18), one obtains the relationship between the resonant frequencies as

$$
f_{n}=\frac{f_{0_{n}}}{\sqrt{1+\alpha_{m} \Delta M}} .
$$

The frequency shift can be expressed using (26) as

$$
\Delta f=f_{0_{n}}-f_{n}=f_{0_{n}}-\frac{f_{0_{n}}}{\sqrt{1+\alpha_{m} \Delta M}} .
$$

From this, we obtain

$$
\frac{\Delta f}{f_{0_{n}}}=1-\frac{1}{\sqrt{1+\alpha_{m} \Delta M}} .
$$

Rearranging gives the expression

$$
\Delta M=\frac{1}{\alpha_{m}\left(1-\left(\Delta f / f_{0_{n}}\right)\right)^{2}}-\frac{1}{\alpha_{m}} .
$$

This equation completely relates the change in mass with the frequency shift. The actual value of the added mass can be obtained from (29) as

$$
M=\frac{\rho A L}{\alpha_{m}} \frac{\left(\alpha_{k}^{2} \beta^{2}\right)}{\left(\alpha_{k} \beta-2 \pi \Delta f\right)^{2}}-\frac{\rho A L}{\alpha_{m}} .
$$

This is the general equation which completely relates the added mass and the frequency shift. The constants appearing in this equation can be summarized as (up to four decimal place) $\alpha_{m}=4, \alpha_{k}=3.5160$ for cantilevered BNNT and $\alpha_{m}=2.5222$, $\alpha_{k}=22.3733$ for bridged BNNT.

To obtain the sensitivity of the BNNT-based sensor, we differentiate (30) with respect to $\Delta f$ and obtain

$$
s=\frac{\partial M}{\partial \Delta f}=\frac{\rho A L}{\alpha_{m}} \frac{4 \pi\left(\alpha_{k}^{2} \beta^{2}\right)}{\left(\alpha_{k} \beta-2 \pi \Delta f\right)^{3}} .
$$

We observe that the mass-detection sensitivity of a BNNT-based nanosensor is a nonlinear function of the frequency shift. For further understanding, expanding the sensitivity in (31) in Taylor series in $\Delta f$, we have

$s=\frac{\rho A L}{\alpha_{m}} \frac{4 \pi}{\left(\alpha_{k} \beta\right)}+\frac{\rho A L}{\alpha_{m}} \frac{24 \pi^{2}}{\left(\alpha_{k} \beta\right)^{2}} \Delta f+\frac{\rho A L}{\alpha_{m}} \frac{96 \pi^{3}}{\left(\alpha_{k} \beta\right)^{3}} \Delta f^{2}+\ldots$

The coefficients associated with the powers of $\Delta f$ are positive. Therefore, the mass sensitivity of the sensor increases with the increasing values of frequency shift $\Delta f$. The minimum sensitivity, for the case when $\Delta f$ is very small, can be obtained by taking $\lim _{\Delta f} \rightarrow 0$ in (32) as

$$
s_{\min }=\frac{\rho A L}{\alpha_{m}} \frac{4 \pi}{\left(\alpha_{k} \beta\right)} .
$$

Applying this equation for the cantilevered and bridged BNNT, the minimum sensitivities can be obtained as

$$
s_{\text {cantilevered }}=\frac{4 \pi}{14.064} \frac{\rho A L}{\beta} \text { and } s_{\text {bridged }}=\frac{4 \pi}{56.430} \frac{\rho A L}{\beta} .
$$

From this, it can be concluded that the cantilevered BNNT sensor is about four times more sensitive compared to the bridged BNNT.

\section{VibRATIONAL ANALYSIS OF BNNT USING MOLECULAR-MECHANICS APPROACH}

Simulations were performed with Gaussian [56], using the universal force field (UFF) developed by Rappe et al. [57]. Since, force fields use explicit expression for the potentialenergy surface of molecule as a function of the atomic coordinates, therefore, force-field-based simulations are convenient. The UFF is well suited for dynamics simulations because it allows more accurate vibration measurements than many other force fields, which do not distinguish bond strengths. The UFF is a purely harmonic force field with a potential-energy expression of the form

$$
\begin{aligned}
E= & \sum E_{R}+\sum E_{\theta}+\sum E_{\phi}+\sum E_{\omega}+\sum E_{\mathrm{VDW}} \\
& +\sum E_{\mathrm{el}} .
\end{aligned}
$$

The valence interactions consist of bond stretching $\left(E_{R}\right)$, which is a harmonic term and angular distortions. The angular distortions are bond angle bending $\left(E_{\theta}\right)$, described by a threeterm Fourier cosine expansion, dihedral angle torsion $\left(E_{\phi}\right)$, and inversion terms (out-of-plane bending) $\left(E_{\omega}\right) . E_{\phi}$ and $E_{\omega}$ are described by cosine-Fourier expansion terms. The nonbonded interactions consist of van der Waals $\left(E_{\mathrm{VDW}}\right)$ and electrostatic $\left(E_{\text {el }}\right)$ terms. $E_{\mathrm{VDW}}$ are described by a Lennard-Jones potential and $E_{\mathrm{el}}$ described by a Coulombic term. The functional form of aforesaid energy terms is given as follows:

$$
\begin{aligned}
E_{R} & =k_{1}\left(r-r_{0}\right)^{2} \\
E_{\theta} & =k_{2}\left(C_{0}+C_{1} \cos \theta+C_{2} \cos 2 \theta\right) \\
C_{2} & =\frac{1}{4 \sin ^{2} \theta} \\
C_{1} & =-4 C_{2} \cos \theta_{0} \\
C_{1} & =C_{2}\left(2 \cos ^{2} \theta_{0}+1\right) \\
E_{\phi} & =k_{3}(1 \pm \cos n \phi) \\
E_{\omega} & =k_{4}\left(1 \pm \cos \left(n \chi-\chi_{0}\right)\right) \\
E_{\mathrm{VDW}} & =D\left[\left(\frac{r^{*}}{r}\right)^{12}-2\left(\frac{r^{*}}{r}\right)^{6}\right] \\
\text { and } E_{\mathrm{el}} & =\frac{q_{i} q_{j}}{\varepsilon r_{i j}}
\end{aligned}
$$

where $k_{1}, k_{2}, k_{3}$, and $k_{4}$ are force constants, $\theta_{0}$ is the natural bond angle, $D$ is the van der Waals' well depth, $r^{*}$ is the van der Waals' length, $q_{i}$ is the net charge of an atom, $\varepsilon$ is the dielectric constant, $r_{i j}$ is the distance between two atoms. The $E_{\text {el }}$ term is a columbic term and is not zero for the present case. The Gaussian [56] program assigned the atomic charges automatically based on the atom types. The atomic charges are assigned according to "Qeq algorithm" presented in [58]. The torsion term $E_{\phi}$ turns out to be of great importance. Detailed values of these parameters 
in (36) can be found in [57]. The calculation of frequency and their validation for CNTs can be found detailed in [59]. In the following section, we are providing the methodology of frequency calculation briefly for completeness of the present paper.

\section{A. Frequency Calculation}

We start with the Hessian matrix $\mathbf{H}_{\mathrm{CAR}}$, which holds the second partial derivatives of the potential $E$ with respect to the displacement of the atoms in cartesian coordinates (CAR) [56], [60]

$$
H_{\mathrm{CAR}_{i j}}=\left(\frac{\partial^{2} E}{\partial \xi_{i} \partial \xi_{j}}\right)_{\mathrm{Opt}}
$$

This is a $3 N \times 3 N$ matrix ( $N$ is the number of atoms), where $\xi_{1}, \xi_{2}, \xi_{3}, \ldots, \xi_{3 N}$ are used for the displacements in cartesian coordinates $\Delta x_{1}, \Delta y_{1}, \Delta z_{1}, \ldots, \Delta z_{N}$. The () Opt refers to the fact that the derivatives are taken at the equilibrium positions of the atoms. These force constants are then converted to mass weighted cartesian (MWC) coordinates [60]

$$
H_{\mathrm{MWC}_{i j}}=\frac{H_{\mathrm{CAR}_{i j}}}{\sqrt{m_{i} m_{j}}}=\left(\frac{\partial^{2} E}{\partial q_{i} \partial q_{j}}\right)_{\mathrm{Opt}}
$$

where $q_{1}=\sqrt{m_{1}} \xi_{1}=\sqrt{m_{1}} \Delta x_{1}, q_{2}=\sqrt{m_{1}} \xi_{2}=\sqrt{m_{1}} \Delta y_{1}$, and so on. $\mathbf{H}_{\mathrm{MWC}}$ is diagonalized, yielding a set of $3 N$ eigenvectors and $3 N$ eigenvalues.

The next step is to translate the center of mass to the origin, and determine the moments and products of inertia, with the goal of finding the matrix that diagonalize the moment of inertia tensor. Using this matrix, we can find the vectors corresponding to the rotations and translations. Once these vectors are known, we know that the rest of the normal modes are vibrations. The center of mass $\mathbf{R}_{\mathrm{COM}}$ is found in the usual way

$$
\mathbf{R}_{\mathrm{COM}}=\frac{\sum_{\alpha} m_{\alpha} \mathbf{r}_{\alpha}}{\sum_{\alpha} m_{\alpha}}
$$

where the sums are over the atoms, $\alpha$. The origin is then shifted to the center of mass $\mathbf{r}_{\mathrm{COM}_{\alpha}}=\mathbf{r}_{\alpha}-\mathbf{R}_{\mathrm{COM}}$. Next, we have to calculate the moments of inertia (the diagonal elements) and the products of inertia (off diagonal elements) of the moment of inertia tensor (I), given by

$\left\{\begin{array}{ccc}\sum_{\alpha} m_{\alpha}\left(y_{\alpha}^{2}+z_{\alpha}^{2}\right) & -\sum_{\alpha} m_{\alpha}\left(x_{\alpha} y_{\alpha}\right) & -\sum_{\alpha} m_{\alpha}\left(x_{\alpha} z_{\alpha}\right) \\ -\sum_{\alpha} m_{\alpha}\left(y_{\alpha} x_{\alpha}\right) & \sum_{\alpha} m_{\alpha}\left(x_{\alpha}^{2}+z_{\alpha}^{2}\right) & -\sum_{\alpha} m_{\alpha}\left(y_{\alpha} z_{\alpha}\right) \\ -\sum_{\alpha} m_{\alpha}\left(z_{\alpha} x_{\alpha}\right) & -\sum_{\alpha} m_{\alpha}\left(z_{\alpha} y_{\alpha}\right) & \sum_{\alpha} m_{\alpha}\left(x_{\alpha}^{2}+x_{\alpha}^{2}\right)\end{array}\right\}$.

This symmetric matrix is diagonalized, yielding the principal moments (the eigenvalues $\mathbf{I}^{\prime}$ ) and a $3 \times 3$ matrix, which is made up of the normalized eigenvectors of (I). The eigenvectors of the moment of inertia tensor are used to generate the vectors corresponding infinitesimal rotation of the molecular system. The translations are trivial to generate in cartesian coordinates. They are just $m_{i}$ times the corresponding coordinate axis. A Schmidt orthogonalization is used to generate $N_{\text {vib }}=3 N-6$ remaining vectors, which are orthogonal to the six rotational and translational vectors. The result is a transformation matrix D which transforms from MWC coordinates $q$ to internal coordinates $\mathbf{S}=\mathbf{D q}$, where rotation and translation have been separated out. Now that we have coordinates in the rotating and translating frames, we need to transform the Hessian $\mathbf{H}_{\mathrm{MWC}}$ to these new internal coordinates (INT) [56], [60]. Only the $N_{\text {vib }}$ coordinates corresponding to internal coordinates will be diagonalized, although the full $3 N$ coordinates are used to transform the Hessian. The transformation is straightforward as follows:

$$
\mathbf{H}_{\mathrm{INT}}=\mathbf{D}^{T} \mathbf{H}_{\mathrm{MWC}} \mathbf{D} \text {. }
$$

The $N_{\text {vib }} \times N_{\text {vib }}$ submatrix of $\mathbf{H}_{\text {INT }}$, which represents the force constants internal coordinates, is diagonalized yielding $N_{\text {vib }}$ eigenvalues and $N_{\text {vib }}$ eigenvectors. If we call the transformation matrix composed of the eigenvectors $\mathbf{L}$, then we have

$$
\mathbf{L}^{T} \mathbf{H}_{\mathrm{INT}} \mathbf{L}=\Gamma
$$

where $\Gamma$ is the diagonal matrix with eigenvalues $\gamma_{i}$. At this point, the eigenvalues need to be converted frequencies as

$$
f_{i}=\sqrt{\frac{\gamma_{i}}{4 \pi^{2}}}
$$

\section{RESULTS AND DISCUSSIONS}

In Section III, it was proved that the cantilever sensor is about four times more sensitive than a bridged sensor. For this reason, in this section, we consider cantilever sensors only. Two types of BNNTs, namely, a armchair and zigzag, with two different lengths are considered. We consider two types of biological objects on the cantilevered BNNT as shown in Fig. 3. Alanine with Amino terminal residue (mass $0.121307 \mathrm{zg}$ ) is a type of $\alpha$-amino acid with simple structure as shown in Fig. 3(a). DeOxy adenosine with free residue (mass $0.416965 \mathrm{zg}$ ) is a nucleoside component of DNA, composed of adenosine and deoxyribose, with a structure shown in Fig. 3(b). Here, we investigate the validity of the mathematical expressions derived in the paper to detect the mass of these molecules. In Fig. 4, the identified masses from the frequency shift of cantilevered BNNTs are shown. Two armchair $(4,4)$ BNNTs of lengths 7.493 and $9.99 \mathrm{~nm}$ are considered. The first BNNT is loaded with upto 13 Alanine with Amino terminal residue molecules shown in Fig. 3(a), while the second BNNT is loaded with upto six DeOxy adenosine with free residue molecules shown in Fig. 3(b). The frequency shift corresponding to these added masses are calculated from the molecular mechanics approach explained in the previous section. These frequency shifts are then used and "experimental results" in the sensor equation (30). The value of the mass predicted by this equation are then compared with the known values used in the molecular mechanics simulations. For 


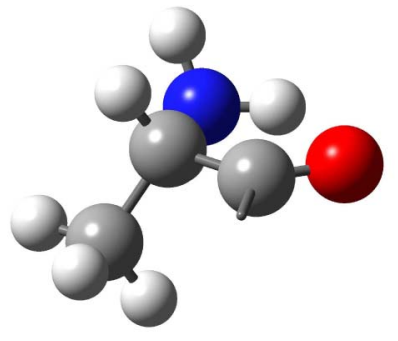

(a)

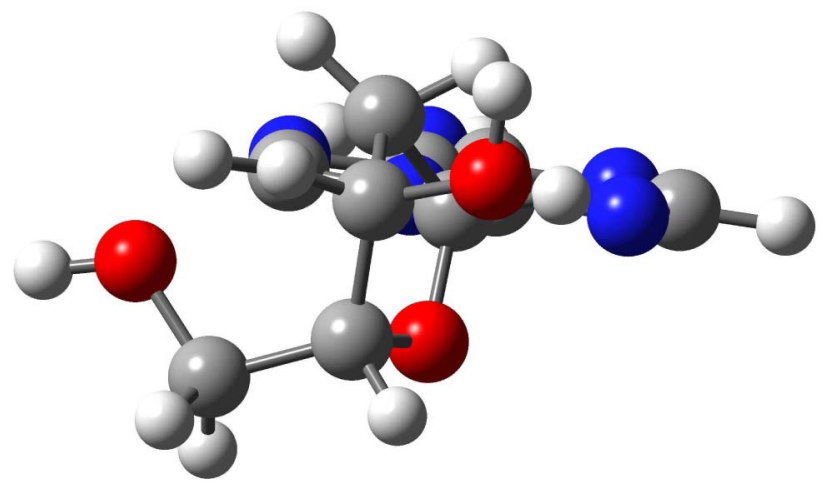

(b)

Fig. 3. Added molecules on the armchair and zigzag BNNTs (1 zg = $10^{-21} \mathrm{gm}$ ). (a) Alanine with Amino terminal residue (mass $0.121307 \mathrm{zg}$ ). The BNNTs are loaded with upto 13 molecules. (b) DeOxy adenosine with free residue (mass $0.416965 \mathrm{zg}$ ). The BNNTs are loaded with upto six molecules.

the BNNT in Fig. 4(a), the predicted mass is very close to the actual mass of the added molecules. By comparing the identified mass with the molecular mechanics simulations in Fig. 4(b), we can see that the sensor equation (30) slightly overpredicts the mass. The general trend is, however, similar. Percentage error obtained using the proposed method is shown in Table I for different values of frequency shift. The error is calculated by considering molecular simulation as the reference. Results for the two cases considered in Fig. 4 are shown. One can observe that the error for the longer BNNT is relatively more than the shorter BNNT. This is possibly due to the fact that the BNNT of length $9.99 \mathrm{~nm}$ is loaded by a much heavier molecule compared to the BNNT of length $7.493 \mathrm{~nm}$.

In Fig. 5, the identified masses from the frequency shift of two zigzag BNNTs are shown. Two zigzag $(5,0)$ BNNTs of lengths 5.191 and $6.922 \mathrm{~nm}$ are considered. Like the armchair BNNTs, the first BNNT is loaded with upto 13 Alanine with Amino terminal residue molecules shown in Fig. 3(a), while the second BNNT is loaded with upto six DeOxy adenosine with free residue molecules shown in Fig. 3(b). The frequency shift corresponding to these added masses are calculated from the molecular- mechanics approach explained in the previous section. The value of the mass predicted by this equation are then compared with the known values used in the molecular mechanics simulations. Percentage error obtained using the proposed method is shown in Table II for different values of frequency

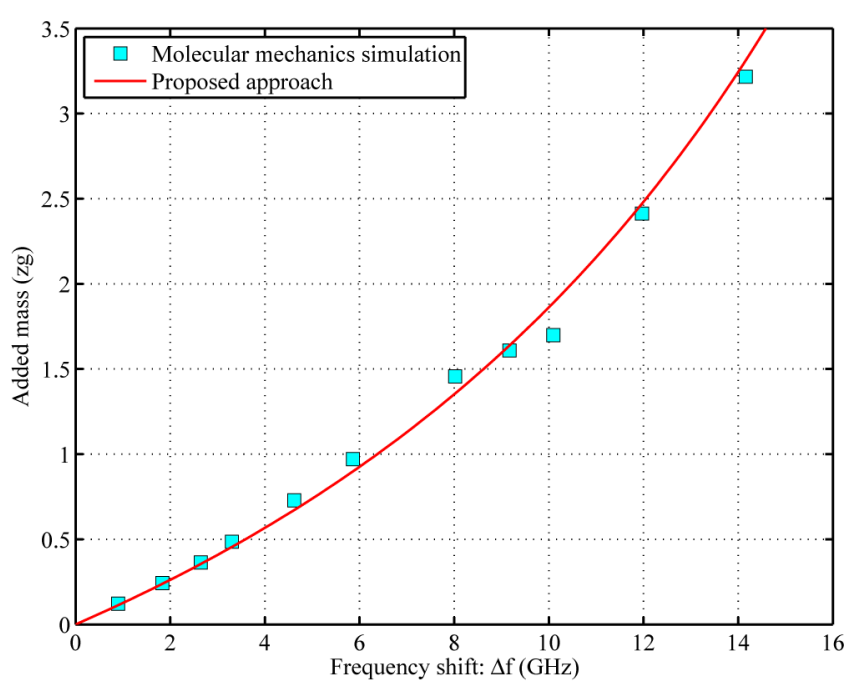

(a)

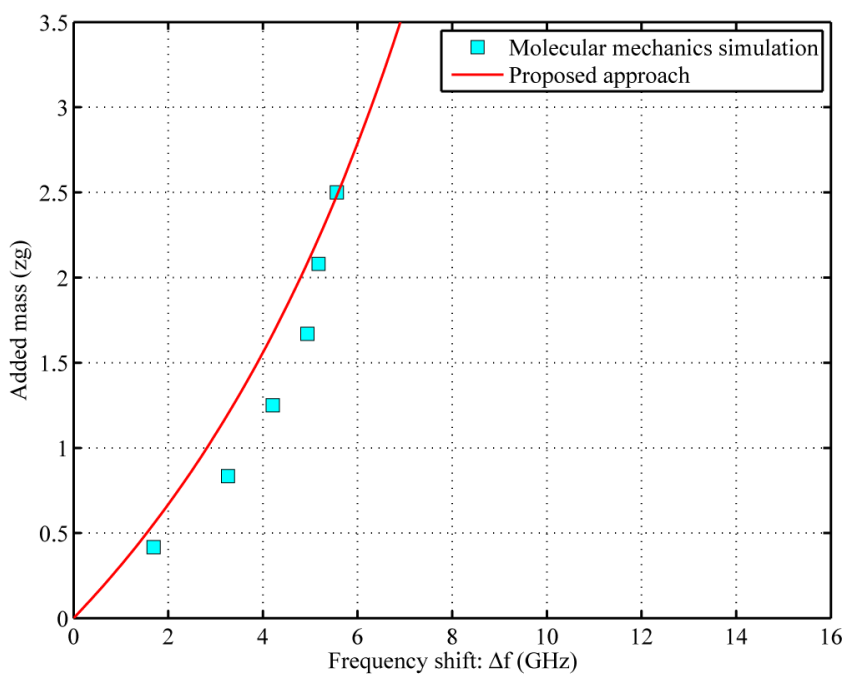

(b)

Fig. 4. Identified attached masses from the frequency shift of a cantilevered armchair BNNT. The proposed analytical approach is validated using data from the molecular mechanics simulations. (a) Alanine with Amino terminal residue on cantilevered armchair $(4,4)$ BNNT. Results of adding upto 13 molecules are shown. The properties of the BNNT are: length $7.493 \mathrm{~nm}$, mass $9.9682 \mathrm{zg}$, diameter $0.551 \mathrm{~nm}$, and fundamental frequency $41.072 \mathrm{GHz}$. (b) DeOxy adenosine with free residue on cantilevered armchair $(4,4)$ BNNT. Results of adding upto six molecules are shown. The properties of the BNNT are: length $9.99 \mathrm{~nm}$, mass $13.2909 \mathrm{zg}$, diameter $0.551 \mathrm{~nm}$, and fundamental frequency $22.847 \mathrm{GHz}$.

TABLE I

Percentage ERror in The Mass Detection Using Cantilevered ARMCHAIR $(4,4)$ BNNT-BASED BIONANOSENSOR

\begin{tabular}{lc}
\multicolumn{3}{l}{$\begin{array}{l}\text { (4,4) BNNT, length } 7.493 \mathrm{~nm} \\
\text { Frequency shift }\end{array}$} \\
\hline (GHz) & \\
\hline 0.8994 & 6.9863 \\
1.8347 & 1.6938 \\
2.6442 & 2.5205 \\
3.3007 & 6.3171 \\
4.6198 & 7.7146 \\
5.8579 & 7.4579 \\
8.0194 & 6.8490 \\
9.1677 & 1.8618 \\
10.0940 & 11.2074 \\
11.9677 & 2.4394 \\
14.1592 & 2.9845
\end{tabular}

$(4,4)$ BNNT, length $9.99 \mathrm{~nm}$ Frequency shift \% error $(\mathrm{GHz})$

\begin{tabular}{ll}
$(\mathrm{GHz})$ \\
\hline 1.6908 & 32.4537
\end{tabular}

$\begin{array}{ll}3.2617 & 43.7511\end{array}$

$4.2091 \quad 33.6174$

$4.9406 \quad 24.9388$

$\begin{array}{ll}5.1744 & 7.2389\end{array}$

$5.5611 \quad 0.7267$ 


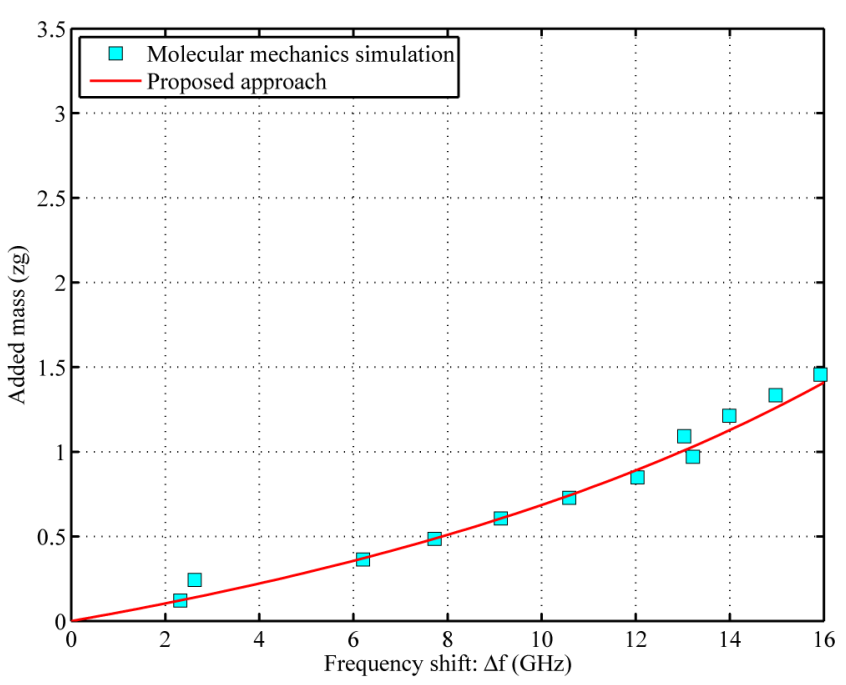

(a)

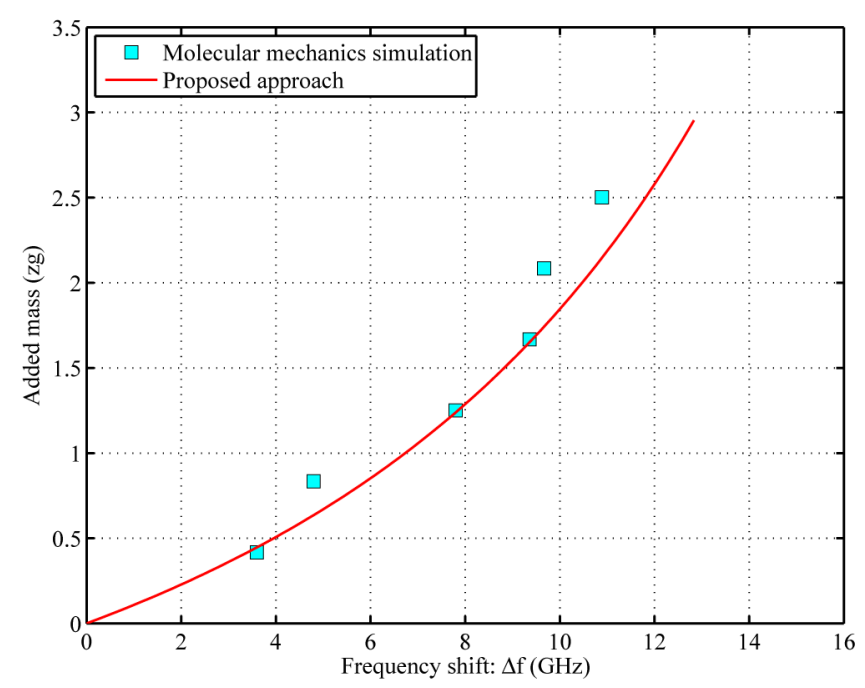

(b)

Fig. 5. Identified attached masses from the frequency shift of a cantilevered zigzag BNNT. The proposed analytical approach is validated using data from the molecular mechanics simulations. (a) DeOxy adenosine with free residue on cantilevered zigzag $(5,0)$ BNNT. Results of adding upto 13 molecules are shown. The properties of the BNNT are: length $5.191 \mathrm{~nm}$, mass $4.98409 \mathrm{zg}$, diameter $0.389 \mathrm{~nm}$, and fundamental frequency $50.80598 \mathrm{GHz}$. (b) Alanine with Amino terminal residue on cantilevered zigzag $(5,0)$ BNNT. Results of adding upto six molecules are shown. The properties of the BNNT are: length $6.922 \mathrm{~nm}$, mass $6.64545 \mathrm{zg}$, diameter $0.389 \mathrm{~nm}$, and fundamental frequency $32.0778 \mathrm{GHz}$.

shift. The error is calculated by considering molecular simulation as the reference. Results for the two cases considered in Fig. 5 are shown. One can observe that the error for both cases are comparable.

The sensitivities of the four cantilevered nanotubes are shown in Table III. These sensitivities are calculated from (34). If identical BNNTs in the bridged configuration were used, their sensitivities would have been $0.0303,0.0403,0.0151$, and $0.0202 \mathrm{zg} / \mathrm{GHz}$, respectively. The cantilevered BNNT are, therefore, more sensitive.
TABLE II

Percentage ERror In The Mass Detection Using CANTILEVEREd ZigZaG $(5,5)$ BNNT BASED BIONANOSENSOR

\begin{tabular}{|c|c|c|c|}
\hline$(5,0)$ BNNT, le & $5.191 \mathrm{~nm}$ & $(5,0)$ BNNT, ler & th $6.922 \mathrm{~nm}$ \\
\hline $\begin{array}{l}\text { Frequency shift } \\
(\mathrm{GHz})\end{array}$ & $\%$ error & $\begin{array}{l}\text { Frequency shift } \\
(\mathrm{GHz})\end{array}$ & $\%$ error \\
\hline 2.3204 & 0.6674 & 3.5975 & 7.0163 \\
\hline 2.6262 & 42.4855 & 4.7967 & 23.7855 \\
\hline 6.2057 & 1.9088 & 7.7946 & 1.0522 \\
\hline 7.7227 & 0.3100 & 9.3595 & 1.0178 \\
\hline 9.1317 & 0.1074 & 9.6653 & 16.4492 \\
\hline 10.5887 & 2.0133 & 10.8885 & 14.2163 \\
\hline 12.0367 & 5.2592 & & \\
\hline 13.2149 & 6.1401 & & \\
\hline 13.0320 & 7.6666 & & \\
\hline 13.9883 & 7.1217 & & \\
\hline 14.9746 & 5.6414 & & \\
\hline 15.9280 & 3.9678 & & \\
\hline 16.6325 & 4.3711 & & \\
\hline
\end{tabular}

TABLE III

SENSITIVITY OF CANTILEVERED BNNT

\begin{tabular}{cccccc}
\multicolumn{7}{c}{ Properties of BNNT } \\
\hline NT type Length & $\begin{array}{l}\text { Mass } \\
(\mathrm{nm})\end{array}$ & \multicolumn{2}{l}{$\begin{array}{l}\text { Diameter Fundamental Sensitivity } \\
(\mathrm{zg})\end{array}$} & $\begin{array}{l}\mathrm{nm}) \\
\text { frequency } \\
(\mathrm{GHz})\end{array}$ & \\
\hline$(\mathrm{zg} / \mathrm{GHz})$ & \\
\hline$(4,4)$ & 7.493 & 9.9682 & 0.551 & 41.072 & 0.1214 \\
$(4,4)$ & 9.990 & 13.2909 & 0.551 & 22.847 & 0.1618 \\
$(5,0)$ & 5.191 & 4.98409 & 0.389 & 50.8059 & 0.0607 \\
$(5,0)$ & 6.922 & 6.64545 & 0.389 & 32.0778 & 0.0809 \\
\hline
\end{tabular}

\section{CONCLUSION}

The use of BNNT as a nanoscale mass sensor is investigated in this theoretical study. The shift in the resonance frequencies due to additional mass is exploited in the proposed sensor. The BNNT resonators are assumed to be either in cantilevered or in bridged configurations. Two atomically configured BNNTs are considered, namely, zigzag and armchair. Simple relationship between the frequency shift and the added mass is proposed, which is based on energy principle. Using this, generalized calibration constants have been derived for an explicit relationship between the added mass and the frequency shift. A molecularmechanics-based approach is used to validate the calibrationconstant-based sensor equations. We used the UFF force field model, wherein the force-field parameters are estimated using the general rules based on the element, its hybridization, and its connectivity. Acceptable agreements between the proposed approach and the molecular mechanics simulations have been observed. Sensitivity equations are also developed. Our results show that BNNTs can be used as biosensor with sensitivity reaching in the order of $0.1 \mathrm{zg} / \mathrm{GHz}$. In addition, it can be noted that the cantilevered BNNT sensor is about four times more sensitive compared to the the bridged BNNT. Theoretical results obtained here may be useful to practically realize future BNNT sensor devices.

\section{REFERENCES}

[1] S. V. Rotkin and S. Subramoney, Applied Physics Carbon Nanotubes: Fundamentals Theory, Optics and Transport Devices. $\quad$ New York: SpringerVerlag, 2005.

[2] T. W. Odom, J. L. Huang, P. Kim, and C. M. Lieber, "Atomic structure and electronic properties of single-walled carbon nanotubes," Nature, vol. 391, no. 6662, pp. 62-64, 1998. 
[3] B. Babic, J. Furer, S. Sahoo, S. Farhangfar, and C. Schonenberger, "Intrinsic thermal vibrations of suspended doubly clamped single-wall carbon nanotubes," Nano Lett., vol. 3, no. 11, pp. 1577-1580, 2003.

[4] V. N. Popov, V. E. Van Doren, and M. Balkanski, "Elastic properties of single-walled carbon nanotubes," Phys. Rev. B, vol. 61, no. 4, pp. 30783084, 2000.

[5] W. H. Duan, C. M. Wang, and Y. Y. Zhang, "Calibration of nonlocal scaling effect parameter for free vibration of carbon nanotubes by molecular dynamics," J. Appl. Phys., vol. 101, no. 2, 2007.

[6] M. Mir, A. Hosseini, and G. H. Majzoobi, "A numerical study of vibrational properties of single-walled carbon nanotubes," Comput. Mater. Sci., vol. 43, no. 3, pp. 540-548, 2008.

[7] S. S. Gupta, F. G. Bosco, and R. C. Batra, "Breakdown of structural models for vibrations of single-wall zigzag carbon nanotubes," J. Appl. Phys., vol. 106, no. 6, 2009.

[8] D. Sanchez-Portal, E. Artacho, J. M. Soler, A. Rubio, and P. Ordejon, "Ab initio structural, elastic, and vibrational properties of carbon nanotubes," Phys. Rev. B, vol. 59, no. 19, pp. 12 678-12 688, 1999.

[9] R. Singh, D. Pantarotto, L. Lacerda, G. Pastorin, C. Klumpp, M. Prato, A. Bianco, and K. Kostarelos, "Tissuenum bio distribution and blood clearance rates of intravenously administered carbon nanotube radio tracers," Proc. Natl. Acad. Sci., vol. 103, no. 9, pp. 3357-3362, 2006.

[10] K. Jensen, K. Kim, and A. Zettl, "An atomic-resolution nanomechanical mass sensor," Nature Nanotechnol., vol. 3, no. 9, pp. 533-537, 2008.

[11] K. Balasubramanian and M. Burghard, "Biosensors based on carbon nanotubes," Anal. Bioanal. Chem., vol. 385, no. 3, pp. 452-468, 2006.

[12] B. L. Allen, P. D. Kichambare, and A. Star, "Carbon nanotube field-effecttransistor-based biosensors," Adv. Mater, vol. 19, no. 11, pp. 1439-1451, 2007.

[13] R. Chowdhury, S. Adhikari, and J. Mitchell, "Vibrating carbon nanotube based bio-sensors," Phys. E: Low-Dimensional Sys. Nanostruct., vol. 42, no. 2, pp. 104-109, Dec. 2009.

[14] C. Y. Li and T. W. Chou, "Mass detection using carbon nanotube-based nanomechanical resonators," Appl. Phys. Lett., vol. 84, no. 25, pp. 52465248, 2004.

[15] X. Blase, A. Rubio, S. Louie, and M. L. Cohen, "Stability and band-gap constancy of boron-nitride nanotubes," Europhysics Lett., vol. 28, no. 5, pp. 335-340, 1994.

[16] N. Chopra, R. Luyken, K. Cherrey, V. Crespi, M. Cohen, S. Louie, and A. Zettl, "Boron-nitride nanotubes," Science, vol. 269, no. 5226, pp. 966 967, 1995.

[17] D. Golberg, Y. Bando, C. Tang, and C. Zhi, "Boron nitride nanotubes," Adv. Mater, vol. 19, no. 18, pp. 2413-2432, 2007.

[18] C. Y. Won and N. R. Aluru, "Structure and dynamics of water confined in a boron nitride nanotube," J. Phys. Chem. C, vol. 112, no. 6, pp. 1812-1818, 2008.

[19] M. Santosh, P. K. Maiti, and A. K. Sood, "Elastic properties of boron nitride nanotubes and their comparison with carbon nanotubes," J. Nanosci. Nanotechnol., vol. 9, no. 9, pp. 5425-5430, 2009.

[20] J. Yuan and K. M. Liew, "Effects of boron nitride impurities on the elastic properties of carbon nanotubes," Nanotechnology, vol. 19, no. 44, 2008.

[21] F. Xu, Y. Bando, D. Golberg, R. Ma, Y. Li, and C. Tang, "Elastic deformation of helical-conical boron nitride nanotubes," J. Chem. Phys., vol. 119 no. 6, pp. 3436-3440, 2003.

[22] W. Moon and H. Hwang, "Molecular mechanics of structural properties of boron nitride nanotubes," Phys. E-Low-Dimensional Sys. Nanostruct., vol. 23, no. 1-2, pp. 26-30, 2004.

[23] V. Pokropivny, S. Kovrygin, V. Gubanov, R. Lohmus, A. Lohmus, and U. Vesi, "Ab-initio calculation of Raman spectra of single-walled BN nanotubes," Phys. E: Low-Dimensional Sys. Nanostruct., vol. 40, no. 7 , pp. 2339-2342, 2008.

[24] J. Song, J. Wu, Y. Huang, K. C. Hwang, and H. Jiang, "Stiffness and thickness of boron-nitride nanotubes," J. Nanosci. Nanotechnol., vol. 8, no. 7, pp. 3774-3780, 2008.

[25] V. Verma, V. K. Jindal, and K. Dharamvir, "Elastic moduli of a boron nitride nanotube," Nanotechnology, vol. 18, no. 43, 2007.

[26] C. Li and T. Chou, "Static and dynamic properties of single-walled boron nitride nanotubes," J. Nanosci. Nanotechnol., vol. 6, no. 1, pp. 54-60, 2006.

[27] L. Wirtz, A. Rubio, R. A. de la Concha, and A. Loiseau, "Ab initio calculations of the lattice dynamics of boron nitride nanotubes," Phys. Rev. B, vol. 68, no. 4, p. 045425, 2003.

[28] G. S. Jeon and G. D. Mahan, "Lattice vibrations of a single-wall boron nitride nanotube," Phys. Rev. B, vol. 79, no. 8, 2009.
[29] H. M. Ghassemi and R. S. Yassar, "On the mechanical behavior of boron nitride nanotubes," Appl. Mech. Rev., vol. 63, no. 2, 2010.

[30] A. Suryavanshi, M. Yu, J. Wen, C. Tang, and Y. Bando, "Elastic modulus and resonance behavior of boron nitride nanotubes," Appl. Phys. Lett., vol. 84, no. 14, pp. 2527-2529, 2004.

[31] N. Chopra and A. Zettl, "Measurement of the elastic modulus of a multiwall boron nitride nanotube," Solid State Commun., vol. 105, no. 5, pp. 297-300, 1998.

[32] C. Zhi, Y. Bando, C. Tang, S. Honda, H. Kuwahara, and D. Golberg, "Boron nitride nanotubes/polystyrene composites," J. Mater. Res., vol. 21, no. 11, pp. 2794-2800, 2006.

[33] Q. Huang, Y. Bando, X. Xu, T. Nishimura, C. Zhi, C. Tang, F. Xu, L. Gao, and D. Golberg, "Enhancing superplasticity of engineering ceramics by introducing BN nanotubes," Nanotechnology, vol. 18, no. 48, 2007.

[34] C. Y. Zhi, Y. Bando, C. C. Tang, Q. Huang, and D. Golberg, "Boron nitride nanotubes: Functionalization and composites," J. Mater. Chem., vol. 18, no. 33, pp. 3900-3908, 2008

[35] D. Golberg, Y. Bando, K. Kurashima, and T. Sato, "Synthesis and characterization of ropes made of BN multiwalled nanotubes," Scr. Mater. vol. 44, no. 8-9, pp. 1561-1565, 2001.

[36] G. Ciofani, V. Raffa, A. Menciassi, and A. Cuschieri, "Boron nitride nanotubes: An innovative tool for nanomedicine," Nano Today, vol. 4, no. 1, pp. 8-10, 2009.

[37] E. S. Oh, "Elastic properties of boron-nitride nanotubes through the continuum lattice approach," Mater. Lett., vol. 64, no. 7, pp. 859-862, 2010.

[38] C. Zhi, Y. Bando, C. Tang, and D. Golberg, "Immobilization of proteins on boron nitride nanotubes," J. Am. Chem. Soc., vol. 127, no. 49, pp. 17144 17145,2005

[39] G. Ciofani, V. Raffa, A. Menciassi, and P. Dario, "Preparation of boron nitride nanotubes aqueous dispersions for biological applications," $J$. Nanosci. Nanotechnol., vol. 8, no. 12, pp. 6223-6231, 2008

[40] G. Ciofani, S. Danti, D. D’Alessandro, S. Moscato, and A. Menciassi, "Assessing cytotoxicity of boron nitride nanotubes: Interference with the MTT assay," Biochem. Biophys. Res. Commun., vol. 394, no. 2, pp. 405411, 2010.

[41] S. K. Georgantzinos and N. K. Anifantis, "Carbon nanotube-based resonant nanomechanical sensors: A computational investigation of their behavior," Phys. E: Low-Dimensional Sys. Nanostruct., vol. 42, no. 5, pp. 1795-1801, 2010.

[42] E. Gil-Santos, D. Ramos, A. Jana, M. Calleja, A. Raman, and J. Tamayo, "Mass sensing based on deterministic and stochastic responses of elastically coupled nanocantilevers," Nano Lett., vol. 9, no. 12, pp. 4122-4127, 2009.

[43] S. Adhikari and R. Chowdhury, "The calibration of carbon nanotube based bio-nano sensors," J. Appl. Phys., vol. 107, no. 12, pp. 124 322:1-8, 2010.

[44] L.-C. Jiang and W.-D. Zhang, "A highly sensitive nonenzymatic glucose sensor based on cuo nanoparticles-modified carbon nanotube electrode," Biosens. Bioelectron., vol. 25, no. 6, pp. 1402-1407, 2010.

[45] S. B. Tooski, "Functionalized single wall carbon nanotube sensor in a perturbed microwave resonant cavity based toxin/pollutant gas pressure sensor," J. Appl. Phys., vol. 107, no. 3, 2010.

[46] E. Hernández, C. Goze, P. Bernier, and A. Rubio, "Elastic properties of c and bxcynz composite nanotubes," Phys. Rev. Lett., vol. 80, no. 20, pp. 4502-4505, 1998.

[47] D. Sánchez-Portal and E. Hernández, "Vibrational properties of singlewall nanotubes and monolayers of hexagonal BN," Phys. Rev. B, vol. 66, no. 23, p. 235415, 2002.

[48] D. Wu, W. Chien, C. Chen, and H. Chen, "Resonant frequency analysis of fixed-free single-walled carbon nanotube-based mass sensor," Sens Actuators A-Phys., vol. 126, no. 1, pp. 117-121, 2006.

[49] C. Wang, C. Ru, and A. Mioduchowski, "Free vibration of multiwall carbon nanotubes," J. Appl. Phys., vol. 97, no. 11, 2005.

[50] F. Scarpa and S. Adhikari, "A mechanical equivalence for the Poisson's ratio and thickness of C-C bonds in single wall carbon nanotubes," $J$. Phys. D: Appl. Phys., vol. 41, no. 085306, pp. 1-5, 2008.

[51] R. Chowdhury, C. Y. Wang, and S. Adhikari, "Low frequency vibration of multiwall carbon nanotubes with heterogeneous boundaries," J. Phys. D: Appl. Phys., vol. 43, no. 085405, pp. 1-8, 2010.

[52] R. D. Blevins, Formulas for Natural Frequency and Mode Shape. Malabar, FL: Krieger Publishing Company, 1984.

[53] L. Meirovitch, Principles and Techniques of Vibrations. New Jersey: Prentice-Hall Inc., 1997.

[54] Y. Li, X. Qiu, F. Yang, X.-S. Wang, and Y. Yin, "Ultra-high sensitivity of super carbon-nanotube-based mass and strain sensors," Nanotechnology, vol. 19 , no. 16,2008 
[55] Y. Yang, C. Callegari, X. Feng, K. Ekinci, and M. Roukes, "Zeptogramscale nanomechanical mass sensing," Nano Lett., vol. 6, no. 4, pp. 583586, 2006.

[56] M. J. Frisch, G. W. Trucks, H. B. Schlegel, G. E. Scuseria, M. A. Robb, J. R. Cheeseman, G. Scalmani, V. Barone, B. Mennucci, G. A. Petersson, H. Nakatsuji, M. Caricato, X. Li, H. P. Hratchian, A. F. Izmaylov, J. Bloino, G. Zheng, J. L. Sonnenberg, M. Hada, M. Ehara, K. Toyota, R. Fukuda, J. Hasegawa, M. Ishida, T. Nakajima, Y. Honda, O. Kitao, H. Nakai, T. Vreven, J. A. Montgomery, Jr., J. E. Peralta, F. Ogliaro, M. Bearpark, J. J. Heyd, E. Brothers, K. N. Kudin, V. N. Staroverov, R. Kobayashi, J. Normand, K. Raghavachari, A. Rendell, J. C. Burant, S. S. Iyengar, J. Tomasi, M. Cossi, N. Rega, J. M. Millam, M. Klene, J. E. Knox, J. B. Cross, V. Bakken, C. Adamo, J. Jaramillo, R. Gomperts, R. E. Stratmann, O. Yazyev, A. J. Austin, R. Cammi, C. Pomelli, J. W. Ochterski, R. L. Martin, K. Morokuma, V. G. Zakrzewski, G. A. Voth, P. Salvador, J. J. Dannenberg, S. Dapprich, A. D. Daniels, Ö. Farkas, J. B. Foresman, J. V. Ortiz, J. Cioslowski, and D. J. Fox, "Gaussian 09 Revision A.1," Gaussian Inc. Wallingford CT, 2009.
[57] A. K. Rappe, C. J. Casewit, K. S. Colwell, W. A. Goddard, and W. M. Skiff, "UFF, a full periodic-table force-field for molecular mechanics and molecular-dynamics simulations," J. Am. Chem. Soc., vol. 114, no. 25, pp. $10024-10035,1992$.

[58] A. K. Rappe and W. A. Goddard, "Charge equilibration for moleculardynamics simulations," J. Phys. Chem., vol. 95, no. 8, pp. 3358-3363, 1991.

[59] R. Chowdhury, S. Adhikari, C. Y. Wang, and F. Scarpa, "A molecular mechanics approach for the vibration of single walled carbon nanotubes," Comput. Mater. Sci., vol. 48, no. 4, pp. 730-735, 2010.

[60] D. F. McIntosh, "The determination of Wilson-Decius F matrix elements from cartesian force constants," Theor. Chem. Acc.: Theory, Comput., Model., vol. 125, no. 3-6, pp. 177-184, 2010.

Author's photographs and biographies not available at the time of publication. 